\title{
Réforme de la première année des études de santé : mise en place d'un outil pour déterminer les étudiants admis dans chaque filière
}

\author{
Reorganizing the first year in health studies: implementation of a tool \\ designed to determine student admissions into each track
}

\author{
Vincent MORICE ${ }^{1}$, André AURENGO ${ }^{1}$ et Emmanuel DURAND ${ }^{2}$ \\ ${ }^{1}$ Faculté de Médecine Pierre et Marie Curie, site Pitié-Salpêtrière, 91 boulevard de l'Hôpital, 75013 Paris, France \\ ${ }^{2}$ Faculté de Médecine Paris-Sud, 63 rue Gabriel Péri, 94276 Le Kremlin-Bicêtre Cedex, France
}

Manuscrit reçu le 17 octobre 2010 ; commentaires éditoriaux formulés aux auteurs le 11 novembre 2011 et le 13 février 2012 ; accepté pour publication le 15 février 2012

\begin{abstract}
Mots-clés
Réforme des études de santé ; concours ; admissions dans les filières ; logiciel de gestion des résultats
\end{abstract}

\section{Keywords}

Reform of health studies; ranking exam; admissions in tracks; results management software
Résumé - Problématique : La réforme de la première année des études de santé définie dans l'arrêté du 28 octobre 2009 a mis en place une année commune à quatre filières (médecine, dentaire, pharmacie, sage-femme). Un concours permet d'obtenir un classement spécifique par filière pour chaque étudiant. La détermination des étudiants admis dans chaque filière doit prendre en compte ces classements et les préférences des étudiants pour chacune d'elle. Objectif : Un algorithme dérivé de celui du mariage stable décrit par Gale et Shapley permet de réaliser cette opération de manière optimale et non discutable. À la Faculté de médecine Pierre et Marie Curie, il a été décidé de développer un logiciel accessible sur Internet, ResExam, qui implémente cet algorithme, permet aux étudiants de définir leurs préférences et sait déterminer leurs classements dans les filières en éliminant les ex-aquo. Résultats : ResExam est un logiciel générique dans lequel le nombre et la description des filières peuvent être adaptés à toute situation (choix de filières, d'options pédagogiques, de stages). Il a été utilisé avec succès par les 2090 étudiants qui se sont présentés au concours en mai 2011 . Il peut être aisément implanté dans d'autres facultés de médecine.

Abstract - Issue: The decree issued on October 28, 2009 reorganized the initial year of health studies by combining four medical study tracks (medicine, odontology, pharmacy, midwifery) in a single year. A competitive examination leads to a specific ranking per track and per student. The selection of students must take into account the ranking as well as student preferences in each track. Objective: An algorithm derived from the Gale and Shapley stability of marriage problem helps carry out this operation optimally and indisputably. The Pierre et Marie Curie school of medicine developed the ResExam software, accessible over internet, that allows students to state their preferences, assigns student rankings in their tracks and eliminates tie scores. 
Results: ResExam is a generic software product that can be suited to any number of students and options (tracks, training courses, etc.). It ran successfully for the 2090 students who passed the competitive examination in May 2011. Other schools of medicine could easily benefit from it.

\section{Introduction et problématique}

Le contexte académique et réglementaire de la première année commune des études de santé (PACES) en France

En France, les étudiants désireux de s'engager dans des études de formation aux métiers de la santé peuvent s'inscrire librement en première année universitaire. Pour les études médicales, odontologiques, pharmaceutiques et maïeutiques, un numerus clausus nécessite la sélection des étudiants par un concours à l'issue de cette première année. Jusqu'en juin 2010, il existait une première année du premier cycle des études médicales (PCEM1) à l'issue de laquelle le concours permettait un classement unique des étudiants inscrits ; les mieux classés choisissaient ensuite entre les études médicales, odontologiques et maïeutiques. De même, il existait une première année du premier cycle des études de pharmacie (PCEP1).

L'arrêté du 28 octobre 2009 relatif à la première année commune aux études de santé (PACES) ${ }^{[1]}$ a mis en place une première année commune aux quatre « filières » (études médicales, odontologiques, pharmaceutiques et maïeutiques), avec un concours unique mais des classements différents par filière. Chaque étudiant peut s'inscrire à tout ou partie de ces filières. Cette réforme s'inscrit dans le cadre de celle du dispositif dit « Licence-Master-Doctorat » (LMD), dans le cadre d'une adaptation aux standards européens ; elle vise aussi à éviter aux étudiants de prolonger plusieurs années leur premier cycle (par exemple deux ans pour tenter le concours de fin de PCEM1 puis, en cas d'échec, deux ans pour tenter le concours de fin de PCEP1), sans avoir de débouché assuré ; des procédures de réorientation ont donc été prévues.
Des dispositions contraignantes et des problèmes à résoudre

L'enseignement de la PACES est donc découpé en unités d'enseignement (UE) affectées d'un certain nombre de crédits (European Credit Transfer System - ECTS). L'année universitaire comprend 7 UE communes (UEC) à toutes les filières et une UE spécifique (UES) par filière choisie par l'étudiant. L'ensemble de l'année représente 90 ECTS et les UES sont enseignées au second semestre. Les coefficients des différentes UE sont établis pour chaque filière.

À la fin du premier semestre, des épreuves portant sur l'enseignement reçu au cours de celui-ci sont organisées et conduisent à plusieurs classements des étudiants : un classement global qui ne repose que sur les UEC affectées de coefficients identiques à leurs nombres d'ECTS et un classement par filière qui utilise les coefficients retenus pour cette filière. À partir de l'année universitaire 2012-2013, les étudiants les moins bien classés dans le classement global pourront être réorientés, dans la limite maximum de $15 \%$ des inscrits. Les autres étudiants doivent choisir la ou les filières dans lesquelles ils vont tenter d'entrer.

À la fin du second semestre, des épreuves portant sur la deuxième partie de l'enseignement permettent d'obtenir de même un classement global portant sur toutes les UEC et un classement spécifique par filière qui prend en compte toutes les UEC et l'UES de cette filière, avec les coefficients propres à la filière. Il est donc nécessaire de gérer cinq classements différents, auxquels s'ajoutent dans certaines universités ceux de filières paramédicales faisant l'objet de conventions particulières. Seuls les étudiants classés en rang utile dans les filières qu'ils ont choisies seront admis à poursuivre leurs études dans l'une de ces filières. À partir de 2011-2012, ceux dont le classement global est au-delà d'un rang compris entre deux fois et demi 
et trois fois le nombre total de places disponibles dans les filières peuvent être réorientés.

Cet arrêté ne précise pas la manière dont les étudiants les mieux classés seront affectés dans les filières. Avant la réforme, à l'issue du concours de la fin de PCEM1, un « amphi de garnison » était organisé : les étudiants étaient appelés tour à tour dans l'ordre de leur classement et indiquaient, dans la limite des numerus clausus, la filière dans laquelle ils voulaient s'engager (médecine, odontologie ou maïeutique). L'introduction d'un classement spécifique par filière rend difficile voire impossible l'utilisation de cette méthode: il n'existe plus d'ordre naturel pour appeler les étudiants et le choix fait par l'un d'eux peut remettre en cause la filière obtenue par un autre.

Afin d'apporter une solution à cette difficulté, nous proposons d'utiliser un algorithme dérivé de l'algorithme du mariage stable proposé par Gale et Shapley ${ }^{[2]}$, qui permet une affectation optimale des étudiants, en prenant en compte les classements dans chaque filière et les préférences des étudiants.

Nous avons donc développé et mis en place à la Faculté de Médecine Pierre et Marie Curie (FMPMC) un logiciel générique accessible sur Internet, ResExam $^{[3]}$, permettant aux étudiants de sélectionner les filières de leur choix tout en indiquant leurs préférences, de calculer leurs rangs à l'issue des épreuves, et de définir lesquels sont réorientés et lesquels sont admis dans chaque filière.

\section{Objectifs du travail}

La présente contribution vise à décrire cette expérience et à en discuter l'intérêt et les limites, en faisant l'hypothèse que les solutions que nous avons mises en œuvre pourraient avoir une portée générale et qu'elles pourraient être transposées à d' autres milieux et à d'autres contextes. Ainsi l'Agence de Mutualisation des Universités et Établissements (AMUE) a mis en place dans le système Apogée certaines procédures analogues $^{[4]}$, en particulier l'algorithme de Gale et Shapley.

\section{Méthode}

\section{L'algorithme du mariage stable}

Cet algorithme vise à apparier un nombre égal d'hommes et de femmes de manière qu' aucun couple extraconjugal ne puisse se former à la satisfaction des deux partenaires. Plus précisément, dans cet algorithme, chaque homme ordonne les femmes selon ses préférences et chaque femme en fait de même avec les hommes. Au départ, tous les hommes et femmes sont libres. Pour chaque homme encore libre, on tente de l'apparier à la femme qui a sa préférence parmi celles auxquelles on n'a pas déjà tenté de l'apparier. Si cette femme est libre, l'appariement (provisoire) a lieu ; sinon l'appariement n'a lieu que si son ancien partenaire n'est pas préféré par la femme; son ancien partenaire redevient alors libre.

Ainsi présenté, cet algorithme favorise les préférences des hommes sur celles des femmes. Si on favorise les préférences des femmes, on peut dans quelques cas très particuliers obtenir des couples différents vérifiant toujours la contrainte de ne pas pouvoir former de couple extraconjugal à la satisfaction des deux partenaires.

Cet algorithme a été présenté en 1962 par Gale et Shapley ${ }^{[2]}$ et utilisé pour le choix de stages hospitaliers aux États-Unis ${ }^{[5]}$ et pour les affectations des étudiants dans le secondaire et le supérieur en Hongrie $^{[6]}$.

Transposition de l'algorithme à la problématique des examens classants multi-filières

Dans le cadre de la PACES, il s'agit d'apparier les étudiants et les filières. Les préférences des filières pour les étudiants reposent sur les classements obtenus pour chacune d'elles. Aucun ex-aquo n'est autorisé par l'algorithme et une procédure pour les séparer doit être prévue. De leur côté, les étudiants indiquent leurs préférences entre les filières. L'algorithme doit être modifié pour prendre en compte le fait que, d'une part, 
chaque filière doit être appariée non pas avec un seul étudiant mais avec un nombre d'étudiants fixé par un numerus clausus (polygamie) et que, d' autre part, certains étudiants ne seront admis nulle part.

Il faut ici se poser la question de savoir qui est favorisé dans l'algorithme, entre les étudiants et les filières. Dans une première version, il nous avait semblé naturel de favoriser les rangs des étudiants : les mieux classés dans une filière y sont $a$ priori admis, quitte à remettre ce choix en cause en fonction des préférences exprimées par les étudiants. Ce choix n'est cependant pas le meilleur dans certains cas très particuliers, comme dans l'exemple suivant. Supposons qu'il n'y ait que deux filières, chacune n'acceptant qu'un étudiant. Supposons aussi qu'un étudiant soit classé premier dans la première, mais que sa préférence va à la seconde où il n'est que second, alors qu'un autre étudiant est classé premier dans la seconde filière alors que sa préférence va à la première où il est second. La première version de l'algorithme conduit à affecter le premier étudiant à la première filière et le second à l'autre, alors que l'inversion des affectations permettrait de respecter leurs préférences. L'algorithme finalement retenu favorise donc les préférences des étudiants sur leurs rangs. Bien que des tests réalisés sur des exemples réalistes n'aient montré aucune différence de résultat entre les deux versions de l'algorithme, favoriser les étudiants est évidemment préférable et c'est la version de l'algorithme retenue dans ResExam.

Il faut également gérer les règles particulières qui s'appliquent à chaque numerus clausus et tout d'abord celle qui stipule que les étudiants venant d'un pays hors Communauté économique européenne (CEE) ne pourront exercer en France et peuvent être acceptés en plus du numerus clausus, sous réserve d'être classés en rang utile et dans la limite de $8 \%$ du numerus clausus initial. D'autres possibilités sont à prévoir, par exemple celle consistant à limiter le nombre d'admis de certaines catégories d'étudiants, même si elles ne sont pas utilisées actuellement.

Enfin il faut prendre en compte le cas d'étudiants qui, bien qu'admis dans une filière, décident $a$ posteriori de se désister. La réutilisation de l'algorithme permet de leur trouver des remplaçants mais doit garantir qu'un étudiant déjà admis dans une filière ne peut être rétrogradé dans une filière de moindre préférence ni, pire, se trouver éliminé. L'utilisation des règles applicables au numerus clausus peut conduire à des situations où l'application directe de l'algorithme n'offre pas cette garantie : une adaptation de l'algorithme s'avère ici nécessaire.

\section{Implémentation de l'algorithme dans un outil générique, ResExam}

La FMPMC a choisi de développer et de mettre en place un outil générique et accessible sur Internet, permettant aux étudiants de se connecter pour indiquer les filières auxquelles ils veulent concourir, ainsi que leurs préférences entre elles, en implémentant l'algorithme de Gale et Shapley pour déterminer les admissions.

Cet outil possède des caractéristiques génériques à plusieurs niveaux :

- Le nombre de filières du concours de la PACES prévu par l'arrêté du 28 octobre 2009 est de quatre. Il peut être augmenté, soit par un changement futur de texte, soit pour les besoins de la faculté. Ainsi, à la FMPMC, une filière complémentaire pour les études de kinésithérapie est prévue; ultérieurement d'autres filières paramédicales y seront peutêtre intégrées. ResExam permet donc de décrire les caractéristiques d'un nombre quelconque de filières, en particulier les UE qui les composent et les informations d'identification à recueillir (nom, redoublant ou non, CEE ou non, etc.).

- L'utilisation de ResExam sur Internet impose une procédure de connexion avec mot de passe pour toutes les opérations sensibles. Il est possible de demander à ResExam de gérer seul les connexions ou de l'intégrer dans l'environnement numérique de travail (ENT) de l'Université et d'utiliser son serveur d'identités dénommé Central Authentication Service $(\mathrm{CAS})^{[7]}$.

- La mise à jour des notes et des informations d'identité des étudiants peut être réalisée soit par import de fichiers de type texte, soit en interactif par le service des examens. Il n'y a pas actuellement de lecture directe des informations dans les bases de données de l'université, en particulier 
l'annuaire. En ce qui concerne la mise à jour interactive, ResExam offre la possibilité de déléguer la saisie à des enseignants pour étendre son champ d'actions à d'autres examens ou concours que la PACES. ResExam peut ici être vu comme un outil collaboratif de saisie; les droits des enseignants sur l'outil sont inférieurs à ceux du service des examens et il est possible de leur cacher certaines informations d'identité ainsi que de gérer des numéros d'anonymat.

- L'automatisation de certains calculs peut-être interdite. Cela concerne les calculs de totaux, des rangs et des admissions dans les filières. Si elles sont utiles, les valeurs non calculées peuvent être intégrées dans les informations recueillies sur les étudiants.

Lorsque les rangs doivent être calculés par ResExam, on peut préciser les règles permettant de séparer les ex-aqquo. Elles peuvent porter sur des informations d'identification (par exemple un redoublant sera prioritaire sur un primant) et/ou sur les UE (par exemple, l'étudiant prioritaire est celui possédant la meilleure note à une UE particulière). Lorsque les règles sont insuffisantes pour départager tous les ex сеquо, ResExam réalise un tirage au sort.

Lorsque les admissions doivent être déterminées par ResExam, il faut définir les numerus clausus et les règles particulières les concernant, par exemple les places autorisées pour les étudiants hors CEE.

- La possibilité pour les étudiants de se connecter pour s'inscrire dans les filières ou donner leurs préférences peut-être ouverte pendant certaines fenêtres de temps uniquement, voire être totalement interdite (le service des examens devra alors mettre lui-même à jour inscriptions et préférences).

- Les données, qu'il s'agisse des informations d'identification, des notes ou des résultats de calculs (totaux, rangs, admissions), peuvent être visualisées par les étudiants, soit globalement, soit limitées à celles de l'étudiant concerné. On n'autorise généralement l'accès aux données qu'aux étudiants qui se sont identifiés et on interdit généralement l'affichage des informations d'identification lors de la visualisation globale.
Outre les affichages, ResExam peut adresser des courriels aux étudiants avec leurs résultats, que ce soit dans des circonstances prédéterminées ou à la demande de la scolarité.

La scolarité dispose quant à elle d'un accès à la totalité des données mais peut de plus sélectionner celles qu'elle veut visualiser et les exporter dans des fichiers.

- ResExam n'est pas destiné à gérer uniquement les résultats d'un concours avec plusieurs filières. Il peut s'adapter à un concours simple ou même à un examen pour lequel la notion de classement et d'admission n'est pas utilisée.

\section{Résultats}

\section{Données générales}

Les caractéristiques décrites ci-dessus et donnant à ResExam son caractère de généricité dérivent pour la plupart de la représentation des concours ou examens choisie dans ResExam.

La gestion simultanée de plusieurs concours ou examens impose en particulier de regrouper ceux qui concernent les mêmes étudiants et de les séparer des autres. La notion de population étudiante a été introduite à cet effet. Les utilisateurs étudiants, enseignants ou de la scolarité sont liés à une (ou plusieurs) population(s) étudiante(s) et n'accèdent qu'aux examens ou concours qu'elle(s) comporte(nt).

Pour représenter un concours possédant plusieurs filières, il faut pouvoir décrire certaines caractéristiques générales du concours, ainsi que des caractéristiques spécifiques à chaque filière. Il s'agit d'une structure hiérarchique dont le tronc porte les informations générales et dont les branches représentent les filières.

Parmi les informations générales, on trouve les noms (court et détaillé) du concours, une date de référence (par exemple l'année concernée), les autorisations d'accès aux résultats, la méthode d'entrée des résultats (import, saisie interactive), les caractéristiques des numéros d'étudiants, la prise en charge 
Tableau I. Schéma de la déclaration du concours de première année commune aux études de santé (PACES).

\section{Population étudiante : PACES}

Examen : concours PACES

Caractéristiques générales : année 2010-2011, notes importées, admissions calculées, etc.

Informations d'identité : nom, primant/redoublant, Communauté économique européenne ou non

Définition de toutes les unités d'enseignement (UE) disponibles

Filière Médecine : totaux et rangs à calculer, règles de séparation des ex aquo, liste des UE concernées avec leurs coefficients, numerus clausus

Autres filières

Réorientation au premier semestre : si le rang est supérieur à $x x x$

Réorientation au second semestre : ne concerne que les étudiants non admis ; les redoublants sont exclus, les primants de rang > yyy sont réorientés, les autres admis à redoubler

ou non de numéros d'anonymat, les codages particuliers utilisables à la place des notes (par exemple «absent ») ou le fait que les admissions doivent être déterminées par le logiciel.

Il faut aussi décrire toutes les informations d'identité qu'on désire recueillir sur les étudiants, ainsi que les UE ou disciplines pour lesquelles des notes seront introduites.

Décrire une filière consiste à définir ses noms court et développé, à indiquer si les totaux et rangs doivent être calculés par le logiciel, à donner le numerus clausus, à préciser les règles permettant de séparer les ex aеquo ou d' agir sur le numerus clausus, etc. Les informations d'identité n'ont pas à être décrites en dehors des caractéristiques générales. En revanche, pour les UE, il faut pouvoir indiquer lesquelles sont concernées par la filière et quel coefficient spécifique appliquer aux notes recueillies.

S'il est prévu que des étudiants puissent être réorientés à l'issue des épreuves, il faut pouvoir décrire les procédures de réorientation si on désire automatiser les décisions. La description d'une stratégie de réorientation est par certains côtés proche de celle d'une filière : noms, calcul automatique ou non de totaux et rangs, règles pour départager les ex áquo, choix des UE intervenant dans les calculs de totaux. Elle doit être complétée par les règles définissant les conditions de réorientation : la procédure s'appliquet-elle avant ou après la détermination des admissions ? Est-elle limitée à une catégorie particulière d'étudiants ? À quelle condition sur les totaux et les rangs un étudiant est-il réorienté ?

Le schéma de la déclaration est résumé dans le tableau I.

\section{Première utilisation opérationnelle de ResExam}

ResExam a été utilisé pour la première fois sur les 2049 étudiants inscrits en PACES à la FMPMC pour l'année universitaire 2010-2011. S'y ajoutent 41 étudiants paramédicaux pour lesquels des numerus clausus spécifiques sont prévus. Ces étudiants paramédicaux ont été gérés comme s'ils se présentaient à un concours indépendant.

Malgré les nombreux tests réalisés avant l'utilisation en grandeur réelle, certaines erreurs sont apparues (par exemple dans le contenu des courriels générés par ResExam), mais ont été corrigées rapidement et n'ont pas remis en cause le fonctionnement. Les étudiants ont pu se connecter sans difficulté depuis leur ENT, que ce soit pour visualiser leurs résultats ou, pendant les périodes autorisées, indiquer les filières où ils voulaient s'inscrire et définir les préférences entre elles. 
Tableau II. Résultats PACES 2011 à la FMPMC.

\begin{tabular}{lccc}
\hline & Inscrits & En premier choix & Admis \\
\hline Médecine & $1889(39)$ & $1486(36)$ & $313(9)$ \\
Odontologie & $774(9)$ & $167(3)$ & $39(0)$ \\
Pharmacie & $639(9)$ & $156(0)$ & $61(0)$ \\
Maïeutique & $464(3)$ & $148(2)$ & $30(0)$ \\
Kinésithérapie & $872(10)$ & $57(0)$ & $26(0)$
\end{tabular}

(Les valeurs entre parenthèses correspondent aux étudiants paramédicaux.)

L'algorithme de détermination des admissions a pu être déclenché aussi souvent que nécessaire pendant la période finale de modification des préférences, puis pendant la période où ont eu lieu les ultimes désistements afin que les étudiants puissent suivre les éventuelles modifications de leurs statuts.

Les résultats finaux, concernant les résultats PACES 2011 à la FMPMC, sont consignés dans le tableau II.

\section{Discussion}

Le logiciel ResExam a été utilisé avec succès sur le concours 2010-2011 de la PACES à la FMPMC. L'algorithme du mariage stable qu'il contient garantit une affectation optimale des étudiants dans les filières prenant en compte à la fois leurs souhaits et leurs rangs, éliminant ainsi toute possibilité de contestation ultérieure.

Les étudiants se sont approprié très facilement les écrans et formulaires qui leur sont proposés, en particulier pour choisir les filières où ils désiraient s'inscrire et définir leurs préférences. En revanche, mis à part les quelques erreurs de jeunesse qu'il a fallu corriger, ils ont été surpris par le fait que la prise en compte de leurs modifications ne se traduisait pas par un recalcul immédiat des affectations dans les filières, ce calcul n'étant déclenché que manuellement plusieurs fois par jour.

Quelques difficultés sont apparues en cours d'utilisation, suite à des demandes qui n'avaient pas été envisagées initialement. Certaines ont été résolues, d'autres non.
- Le concours d'entrée dans la filière kinésithérapie ne permet de valider que 60 ECTS au lieu des 90 auxquels l'année doit théoriquement permettre de prétendre. Il a donc fallu être capable d'indiquer à ResExam qu'un étudiant ne pouvait s'inscrire uniquement à cette filière.

- La prise en charge des paramédicaux comme des étudiants passant un concours indépendant n'est actuellement pas satisfaisante. Il faudrait en effet qu'ils soient classés parmi les autres étudiants mais avec des numerus clausus spécifiques, ce que ResExam ne sait pas gérer.

- La possibilité de réorientation des étudiants en cours d'année ne sera pas utile avant l'année universitaire 2012-2013. Sa mise en place complète dans ResExam est encore à l'étude.

- La gestion des démissions après que les affectations dans les filières aient été réalisées de manière théoriquement définitive est réalisable par ResExam mais c'est au service de la scolarité de déclarer chaque démission, le logiciel se chargeant de proposer un remplaçant. Pour alléger le travail de ce service, il faudra que ResExam autorise des étudiants admis à se connecter directement pour démissionner.

- Suite à une convention entre l'Université de Nouvelle-Calédonie et l'UPMC, un concours de PACES est organisé à Nouméa et les étudiants admis viennent en France poursuivre leurs études. Ce concours est distinct de celui proposé en France, tant sur les sujets que sur les dates. Pour que ResExam puisse gérer ces étudiants, il a fallu leur permettre de s'y connecter en utilisant leur propre serveur $C A S$ de login, tout en laissant le service de scolarité en métropole accéder aux données en se connectant à l'aide du serveur CAS de l'UPMC.

Le logiciel ResExam a été écrit pour être générique, non seulement vis-à-vis de la description des examens et concours, mais aussi pour permettre une diffusion aisée à d' autres universités, et pas nécessairement dans le domaine de la santé. Son utilisation est indépendante des autres logiciels utilisés par les services de scolarité puisque leur intercommunication est assurée par des imports et exports de fichiers de type texte. 
D'un point de vue technique, le logiciel ResExam peut être installé sur n'importe quel serveur HTTP. Il est écrit en PHP (version 5) et utilise le système de gestion de bases de données MySql (version 5). Le serveur doit pouvoir envoyer des courriels. Si ResExam doit utiliser un serveur CAS pour l'identification des utilisateurs, le paquetage CURL doit également être installé sur le serveur.

Le logiciel comporte un fichier pour générer la structure de la base de données, les fichiers de programme PHP, et la documentation ${ }^{[3]}$. Seules quelques variables de configuration décrites dans la documentation doivent être modifiées après l'installation. L'ensemble des fichiers peut être obtenu en contactant V. Morice, Faculté de Médecine Pierre et Marie Curie, Site Pitié-Salpêtrière, 91 Bd de l'Hôpital, 75013 Paris.

\section{Conclusion}

L'outil ResExam nous paraît être une réponse adaptée au problème des examens classants multifilières. Bien qu'il reste quelques éléments à développer, il a déjà démontré sa capacité à prendre en charge avec succès plusieurs milliers d'étudiants et sa faculté d'adaptation à diverses structures d'examens et concours.

\section{Contributions}

Vincent Morice est l'auteur du logiciel ResExam. André Aurengo est l'enseignant responsable de la PACES à la faculté de médecine Pierre et Marie Curie. Emmanuel Durand a validé l'algorithme et le logiciel par plusieurs tests.

\section{Remerciements}

Nous remercions les doyens Serge Uzan (UPMC) et Dominique Porquet (Paris Sud) pour leur aide et leur soutien.

\section{Références}

1. Arrêté du 28 octobre 2009 relatif à la première année commune aux études de santé. Journal officiel de la République Française, 17 novembre 2009; n 0266 :19838. 2009 [On-line] Disponible sur: http://www.legifrance.gouv.fr/ affichTexte.do?cidTexte=JORFTEXT000021276755 \&dateTexte $=$ \&categorieLien $=i d$

2. Gale D, Shapley LS. College Admissions and the Stability of Marriage. Amer Math Monthly 1962;69:914.

3. Morice V. ResExam, un outil de gestion des résultats d'examens, 2011 [On-line]. Disponible sur : http:// resexam.admp6.jussieu.fr/docs/resexam.pdf

4. Agence de mutualisation des universités et établissements (AMUE). Procédure de choix des filières PACES. Note du 20 mai 2010.

5. Irving R, Manlove D. Stable machine algorithms. EPSRC research project GR/M13329 1998-2000 [On line]. Disponible sur: http://www.dcs.gla. ac.uk/research/algorithms/stable/

6. Biro P. Student admission in Hungary as Gale and Shapley envisaged. 2008. Technical report TR-2008291, University of Glasgow, 2008 [On line]. Disponible sur http://www.dcs.gla.ac.uk/publications/ PAPERS/8999/ca_tr.pdf

7. Mathieu V, Aubry P, Marchal J. Single Sign-On opensource avec CAS (Central Authentication Service). Lille, JRES 2003.

Correspondance : Vincent Morice, Faculté de Médecine Pierre et Marie Curie, site Pitié-Salpêtrière, 91 boulevard de l'Hôpital, 75013 Paris, France. Mailto: vincent.morice@upmc.fr 